\title{
Intracanal Cryotherapy and its Effects on the Postoperative Pain in Root Canal Therapy: Systematic Review \& Meta-analysis Protocol.
}

Durre Sadaf ( $\nabla$ d.ahmad@qu.edu.sa )

Qassim University College of Dentistry https://orcid.org/0000-0003-4504-6267

Muhammad Zubair Ahmad

Qassim University College of Dentistry

Igho Onakpoya

University of Oxford

\section{Protocol}

Keywords: canal irrigation, cryotherapy, irreversible pulpitis, postoperative pain, root canal therapy

Posted Date: January 15th, 2020

DOl: https://doi.org/10.21203/rs.2.20935/v1

License: (c) (i) This work is licensed under a Creative Commons Attribution 4.0 International License.

Read Full License 


\section{Abstract}

Background: There are many research studies directing in lowering postoperative pain in root canal therapy. The use of intracanal cryotherapy in root canal treatment is recently introduced, and many researchers are inclined towards this techniques. This systematic review will give an insight into its application in endodontic therapy and its effects on postoperative pain.

The aim of this systematic review is to evaluate the effect of intracanal cryotherapy on the postoperative pain in patients with pulp diseases and with or without peri-radicular infection during root canal therapy.

This systematic review will include randomized controlled trials only. The studies should encompass patients with pulpal diseases, i.e. irreversible pulpitis and pulp necrosis in teeth. Previously root treated teeth, immature, and teeth with root resorption will not be included.

Methods: We will conduct a comprehensive search strategy using electronic databases PubMed, EMBASE (Ovid), Scopus, and Cochrane library. A predefined inclusion and exclusion criteria will be utilized for the selection of studies. The reference list of selected studies will be manually searched. We will extract data from studies on a structured data collection form. At the same time, the risk of bias assessment of studies using the Cochrane risk of bias assessment tools will be performed by two reviewers independently. Furthermore, qualitative and quantitative data synthesis will be accomplished. The data will be presented in the form of table of summary of findings.

Discussion: This systematic review will assess the role of intracanal cryotherapy in the form of cold saline irrigation as a final irrigant during root canal therapy. The quality of evidence will ranked as high, moderate low or very low using GRADE approach. Comprehensive search strategy, risk of bias assessment using Cochrane risk of bias assessment tool by two reviewers are the strength of this systematic review.

Systematic review registration number: The protocol is registered with the International Prospective Register of Systematic Reviews. (PROSPERO CRD 42020163438)

\section{Background}

There is a high prevalence of postoperative pain associated with root canal therapy ranges from $3 \%-58 \%$ (1). Pain is a multifactorial phenomenon. Bacterial, mechanical and chemical influences contribute to postoperative pain in root canal therapy (2). Pulpal and peri radicular conditions, preoperative pain and presence of periapical radiolucency are considered potential contributing factors to postoperative pain during root canal therapy (3-5). Postoperative pain in root canal therapy is very disturbing to both patients and dentists. That is why several research studies are focusing on lowering postoperative pain. These include pharmacological management(6-9), behavioural techniques to reduce patient's anxiety (10), 
application of different instrument with different kinematics (11). With this in mind, management of postoperative pain should be an integral part of endodontic therapy (3).

Cryotherapy is a type of treatment that involves the application of cold in order to reduce pain(12). It has been found to be effective in reducing oedema, swelling, pain and inflammation. Cold application has long been used in many branches of medicine including orthopaedics, physiotherapy, neurology, maxillofacial surgery, plastic surgery, and dental surgery(13-18). An in vitro study reported a decrease in the temperature of the external surface of root by the irrigation of cold saline that may likely to have antiinflammatory effects on the surrounding peri radicular tissues(19).

The mechanism of pain reduction by cold therapy has been investigated in many research studies. It decreases the temperature and causes vasoconstriction that follows a decrease in cell metabolism, and restricts the production of free radicals in tissues. These sequence of events lead to a decrease in pain (20-24). Moreover, thermoreceptors are specialized nerve endings that are triggered by a change in tissue temperature. These receptors are thought to be stimulated by cold application. They constrain nociceptors, thus reducing pain(20).

In dentistry, cold application after oral surgical procedures has been utilized to reduce post-operative pain $(25,26)$.

A preliminary search was conducted in PROSPERO, MEDLINE, and the Cochrane Database of Systematic Reviews and no current or underway systematic review on this topic was identified.

The objective of this systematic review is to evaluate the effect of intracanal cryotherapy in reducing postoperative pain during root canal therapy in patients presented with irreversible pulpitis with or without apical periodontitis.

\section{Review Question}

A well-defined clinical question in PICO (Patient/Problem, Intervention, Comparison, Outcome) format developed:

Is intracanal cryotherapy in the form of cold saline as a final irrigant (Intervention) during root canal therapy effective in reducing postoperative pain (Outcome) as compared to irrigation at room temperature(Comparison) in patients presented with various pulpal diseases with or without apical periodontitis (Population/Problem)?

\section{Eligibility Criteria}

\section{Participants}

The review will include studies in which patients have any pulpal or periapical disease in their teeth, and they need root canal therapy. Previously root treated teeth, teeth with immature apex or teeth with root resorption will not be included. 


\section{Intervention}

Cold saline (2.5 degree Celsius ) as a final irrigant solution after standard chemomechanical preparation of the root canals.

\section{Comparison}

Saline irrigation at room temperature as a final irrigant after standard chemomechanical preparation of root canals.

\section{Outcome}

Postoperative pain from the first day to the seventh day.

\section{Study Designs}

Only Randomized controlled trials will be included for the review. Case reports, observational studies and Animal studies will be excluded.

\section{Methods}

This protocol was developed according to the recommendations of the Preferred Reporting Items for Systematic Review and Meta-Analysis Protocol guidelines (PRISMA-P) (27).

The protocol is registered with the International Prospective Register of Systematic Reviews. (PROSPERO CRD 42020163438)

\section{Search Strategy}

A comprehensive search strategy with the help of an expert librarian will be devised. Keywords, texts words and synonymous primarily conceived from the PICO* framework will be searched from $\mathrm{MeSH}^{*}$ database, previous research articles, and books. PubMed, EMBASE, Scopus and Cochrane library databases will be utilized to searach relevant studies. There will be no time or language limitation. An advanced search strategy on PubMed is conducted for exploring relevant studies as an example. (Annexure-1)

Grey literature and unpublished studies will be searched through Google Scholar and ProQuest.

Reference list of identified studies will be manually checked for identification of further studies. Three endodontic journals: Journal of Endodontics, International Endodontic Journal and, Australian Endodontic Journal will be manually searched from 2000-2019.

\section{Data Extraction.}


All selected studies from various sources will be transported to EndNote Software X9. One reviewer will make an initial screening of studies by titles and abstracts to remove irrelevant and duplicate studies. The full text of the remaining studies will then be assessed by the two reviewers (DS, ZA) using the inclusion and exclusion criteria mentioned above. If there will be any disagreement over the eligibility of any study, it will be resolved through discussion. The whole process of studies selection will be done in a transparent, systematic and reproducible way and will be presented in the PRISMA flow diagram (27).

A standardized data extraction form on an Excel sheet will be created with a consensus of two reviewers (DS, ZA). The data will include study ID, sample size in the intervention and the control groups, diagnosis, type of teeth, concentration of irrigant, characteristics of intervention groups and control groups, preoperative pain, postoperative pain on $1 \mathrm{st}, 2^{\text {nd }}, 3^{\text {rd }}, 4^{\text {th }}, 5^{\text {th }}, 6^{\text {th }}, 7^{\text {th }}$, days and conclusion. Data extraction will be done by the two reviewers (DS, ZA) independently. Before data extraction, the two reviewers will be calibrated in order to achieve consistency in data extraction. Authors of included studies will be contact where necessary information will be needed.

\section{Risk of Bias Assessment}

Quality assessment of studies will be carried out as a part of data extraction using the Cochrane Risk of Bias Assessment Tool. We will evaluate six domains in each study under this tool: Random sequence generation, Allocation concealment, Blinding of participants and operators, Blinding of outcome assessors, incomplete outcome data, and other methodologic bias. The study will be ranked as low risk of bias if all domains are adequately reported in the text, High risk of bias if all or anyone domain is not reported in the text, and Unclear risk of bias if any one of the domain is not described satisfactorily in the text.

\section{Strategies for data synthesis}

We will synthesize the data both quantitatively and qualitatively. In qualitative analysis, various characteristics of included studies will be evaluated and compared. In addition, we will estimate the pooled effect size of postoperative pain in the form of weighted mean difference (WMD) or Standardized mean difference (SMD) (in case of different scales of pain measurements are used). Pooled effect size will be displayed in the form of the forest plot. We will used RevMan 5.3 for statistical analysis of the data.

We are expecting heterogeneity among studies due to variability in study designs, clinical methodology, different types of teeth and variable pulpal and periapical status of teeth, and sampling variability. Heterogeneity among studies will be estimated by $\mathrm{I}^{2}$ statistics and P-value. The degree of heterogeneity will be considered acceptable if $\mathrm{I}^{2}<30 \%$, Moderate heterogeneity if $\mathrm{I}^{2}$ is $30-60 \%$, and substantial heterogeneity if $\mathrm{I}^{2}>60 \%$.

Due to the anticipated heterogeneity among study, random-effect model will be used for meta-analysis. A random-effect model allows variability in study designs, methodology and especially sampling 
variability. Sensitivity analysis will also be conducted in order to evaluate the robustness of results from the meta-analysis.

\section{Subgroup analysis \& Publication bias.}

If we found a substantial heterogeneity, the reasons behind the heterogeneity will be explored. All possible factors responsible for heterogeneity may be further divided into subgroups. Subgroup analysis will be performed to evaluate heterogeneity. Publication bias within studies will be assessed by funnel plot.

\section{Discussion}

The primary outcome of the treatment effect will be presented in the form of a table of summary of findings. The quality of evidence will be evaluated by using the Grading of Recommendation, Assessment, Development, and Evaluation (GRADE) approach. The quality of evidence for each outcome will be rated as high, moderate, low and very low. This review will guide clinicians to help in controlling postoperative pain during root canal therapy in a predictable and effective way. It will also direct a new avenue for researchers as well.

\section{Declarations}

\section{Abbreviations}

PICO: Patients/Population, Intervention, Comparison, Outcome

MeSH: Medical Sub-Headings

Ethical Approval and Consent to participate

Not applicable

Availability of supporting data

Yes

\section{Competing Interest}

None

\section{Funding}

This systematic review is not funded by any public or commercial agency.

Contribution Of Authors 
This systematic review will be contributed by Durre Sadaf, Muhammad Zubair Ahmad and Igho Onakpoya. Durre Sadaf is the corresponding author and principle investigator. Muhammad Zubair Ahmad is coinvestigator and contribute in writing, and revision of the review. Igho Onakpoya is methodological expert and contribute in writing and revision. All authors approved the final manuscript.

\section{Acknowledgement}

Not applicable

\section{Authors' Information}

1. Corresponding Author: Durre Sadaf

Assistant Professor, Department of Conservative Sciences, Qassim University, Buraidah, KSA.

Evidence-Based Healthcare, Centre of Evidence-Based Medicine, University of Oxford, UK.

Mailing Address: C-702, La Palma Qassim Housing Compound West Ring Road, Al-Humr Exit】 BURAYDHA®QASSIM®51451囚SAUDI ARABIA.Email: durre.sadaf@hertford.ox.ac.uk

\section{d.ahmad@qu.edu.sa}

Tel: +966590379192

2. Muhammad Zubair Ahmad:

Assistant Professor, Department of Conservative Sciences, Al Rass, Qassim University, KSA.

Evidence-Based Healthcare, Centre of Evidence-Based Medicine, University of Oxford, UK.

Email.zubair.ahmad@hertford.ox.ac.uk

Tel: +966582527047

3. Igho Onakpoya

University of Oxford, Centre for Evidence-Based Medicine, Nuffield Department of Primary Care Health Sciences, Woodstock Road, Oxford OX2 6GG

Email: igho.onakpoya@phc.ox.ac.uk

Tel: +44 (0) 1865289672

\section{References}


1. Sathorn $\mathrm{C}$, Parashos $\mathrm{P}$, Messer $\mathrm{H}$. The prevalence of postoperative pain and flare-up in single- and multiple-visit endodontic treatment: a systematic review. International endodontic journal. 2008;41(2):91-9.

2. Matusow RJ. The flare-up phenomenon in endodontics: A clinical perspective and review. Oral Surgery, Oral Medicine, Oral Pathology. 1988;65(6):750-3.

3. Ali A, Olivieri JG, Duran-Sindreu F, Abella F, Roig M, Garcia-Font M. Influence of preoperative pain intensity on postoperative pain after root canal treatment: A prospective clinical study. Journal of dentistry. 2016;45:39-42.

4. Ince B, Ercan E, Dalli M, Dulgergil CT, Zorba YO, Colak H. Incidence of postoperative pain after singleand multi-visit endodontic treatment in teeth with vital and non-vital pulp. Eur J Dent. 2009;3(4):2739.

5. Ng YL, Glennon JP, Setchell DJ, Gulabivala K. Prevalence of and factors affecting post-obturation pain in patients undergoing root canal treatment. International endodontic journal. 2004;37(6):38191.

6. Attar S, Bowles WR, Baisden MK, Hodges JS, McClanahan SB. Evaluation of Pretreatment Analgesia and Endodontic Treatment for Postoperative Endodontic Pain. Journal of Endodontics. 2008;34(6):652-5.

7. Rowe NH, Shekter MA, Turner JL, Spencer J, Dowson J, Petrick TJ. Control of pain resulting from endodontic therapy: A double-blind, placebo-controlled study. Oral Surgery, Oral Medicine, Oral Pathology. 1980;50(3):257-63.

8. Torabinejad M, Dorn SO, Eleazer PD, Frankson M, Jouhari B, Mullin RK, et al. Effectiveness of various medications on postoperative pain following root canal obturation. Journal of Endodontics. 1994;20(9):427-31.

9. Ryan JL, Jureidini B, Hodges JS, Baisden M, Swift JQ, Bowles WR. Gender Differences in Analgesia for Endodontic Pain. Journal of Endodontics. 2008;34(5):552-6.

10. Gatchel RJ. Dealing with Discomfort Managing Anxiety and Pain During Dental Treatment. The Journal of the American Dental Association. 1992;123(6):37-41.

11. Arslan $H$, Khalilov R, Do anay $E$, Karatas $E$. The effect of various kinematics on postoperative pain after instrumentation: a prospective, randomized clinical study. Journal of Applied Oral Science. 2016;24:503-8.

12. Dehghan M, Farahbod F. The efficacy of thermotherapy and cryotherapy on pain relief in patients with acute low back pain, a clinical trial study. J Clin Diagn Res. 2014;8(9):LC01-LC4.

13. Bleakley C, McDonough S, MacAuley D. The use of ice in the treatment of acute soft-tissue injury: a systematic review of randomized controlled trials. The American journal of sports medicine. 2004;32(1):251-61.

14. Grant AE. MASSAGE WITH ICE (CRYOKINETICS) IN THE TREATMENT OF PAINFUL CONDITIONS OF THE MUSCULOSKELETAL SYSTEM. Archives of physical medicine and rehabilitation. 1964;45:233-8. 
15. Diamond S, Freitag FG. Cold as an adjunctive therapy for headache. Postgraduate medicine. 1986;79(1):305-9.

16. Belli E, Rendine G, Mazzone N. Cold therapy in maxillofacial surgery. The Journal of craniofacial surgery. 2009;20(3):878-80.

17. Akan M, Misirlioglu A, Yildirim S, Cakir B, Taylan G, Akoz T. Ice application to minimize pain in the split-thickness skin graft donor site. Aesthetic plastic surgery. 2003;27(4):305-7.

18. Forouzanfar T, Sabelis A, Ausems S, Baart JA, van der Waal I. Effect of ice compression on pain after mandibular third molar surgery: a single-blind, randomized controlled trial. International journal of oral and maxillofacial surgery. 2008;37(9):824-30.

19. Vera J, Ochoa-Rivera J, Vazquez-Carcano M, Romero M, Arias A, Sleiman P. Effect of Intracanal Cryotherapy on Reducing Root Surface Temperature. J Endod. 2015;41(11):1884-7.

20. Nadler SF, Weingand K, Kruse RJ. The physiologic basis and clinical applications of cryotherapy and thermotherapy for the pain practitioner. Pain physician. 2004;7(3):395-9.

21. Lee H, Natsui H, Akimoto T, Yanagi K, Ohshima N, Kono I. Effects of cryotherapy after contusion using real-time intravital microscopy. Medicine and science in sports and exercise. 2005;37(7):10938.

22. Dolan MG, Thornton RM, Fish DR, Mendel FC. Effects of cold water immersion on edema formation after blunt injury to the hind limbs of rats. J Athl Train. 1997;32(3):233-7.

23. McLean DA. The use of cold and superficial heat in the treatment of soft tissue injuries. Br J Sports Med. 1989;23(1):53-4.

24. Muldoon J. Skin cooling, pain and chronic wound healing progression. British journal of community nursing. 2006;11(3):S21, s4-5.

25. Forsgren $H$, Heimdahl A, Johansson B, Krekmanov L. Effect of application of cold dressings on the postoperative course in oral surgery. International journal of oral surgery. 1985;14(3):223-8.

26. Laureano Filho JR, de Oliveira e Silva ED, Batista $\mathrm{Cl}$, Gouveia FM. The influence of cryotherapy on reduction of swelling, pain and trismus after third-molar extraction: a preliminary study. Journal of the American Dental Association (1939). 2005;136(6):774-8; quiz 807.

27. Moher D, Shamseer L, Clarke M, Ghersi D, Liberati A, Petticrew M, et al. Preferred reporting items for systematic review and meta-analysis protocols (PRISMA-P) 2015 statement. Syst Rev. 2015;4:1.

\section{Supplementary Files}

This is a list of supplementary files associated with this preprint. Click to download.

- Search.startegydocx.docx

- PRISMAPchecklist.docx 